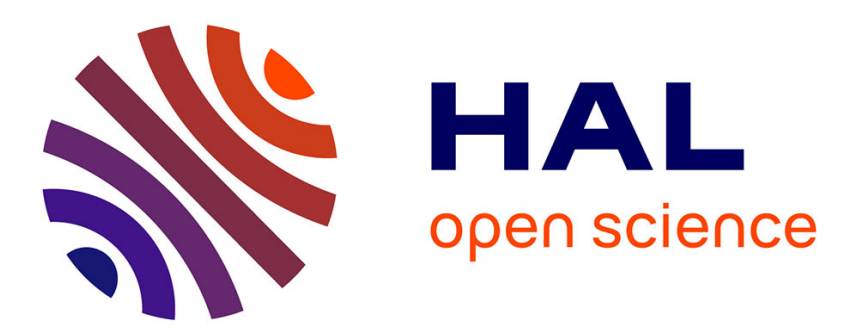

\title{
Soigner la psychose. Le cadre de soin extrahospitalier : un objet à utiliser
}

Cécile Luong, Derek Humphreys

\section{To cite this version:}

Cécile Luong, Derek Humphreys. Soigner la psychose. Le cadre de soin extrahospitalier : un objet à utiliser. L'Évolution Psychiatrique, 2010, 75 (3), pp.485-497. 10.1016/j.evopsy.2010.05.001 . hal02523955

\section{HAL Id: hal-02523955 \\ https://hal.science/hal-02523955}

Submitted on 30 Mar 2020

HAL is a multi-disciplinary open access archive for the deposit and dissemination of scientific research documents, whether they are published or not. The documents may come from teaching and research institutions in France or abroad, or from public or private research centers.
L'archive ouverte pluridisciplinaire HAL, est destinée au dépôt et à la diffusion de documents scientifiques de niveau recherche, publiés ou non, émanant des établissements d'enseignement et de recherche français ou étrangers, des laboratoires publics ou privés. 
L'évolution psychiatrique 75 (2010) 485-497

\title{
Corps et psychose \\ Soigner la psychose. Le cadre de soin extrahospitalier : un objet à utiliser
}

\author{
Care of psychotic patients. The outpatient care setting: \\ The use of an object \\ Cécile Luong, Psychologue clinicienne \\ Derek Humphreys, MD, PHD, Laboratoire de psychologie \\ clinique et psychopathologie, université Paris-Descartes
}

L'intérêt de cet article réside en ce qu'il puise à une expérience auprès de patients en grande souffrance psychique et dans la nécessité de réfléchir à une mise en mots de ce qui se joue dans l'interrelation malade/soignants articulée à un cadre de soin extra-hospitalier. C'est peut-être dans l'écart où ce type de structure spécifique se propose d'intervenir et dans ce qu'il comporte d'extrêmement stimulant et de subtilement exploitable pour la dynamique psychique des patients, que pourrait être envisagé un questionnement du modèle d'intervention de la psychiatrie actuelle.

Pour étayer notre démonstration, nous reprendrons le chemin parcouru par les pionniers de l'approche psychanalytique de la psychose. On pourrait dire tout d'abord que la réflexion ouverte par Freud, à partir du cas de paranoïa du Président Schreiber, s'appuyait sur l'idée d'une perturbation de la relation libidinale d'un sujet à la réalité, et sur l'importance d'y lire la reconnaissance d'un insoutenable projeté hors-soi mais renvoyant à l'intérieur, au psychique. Mélanie Klein, pour appuyer sa thèse d'un clivage originaire, introduisit grâce à ses observations et analyses d'enfants, une relation d'objet précoce, le repérage d'un indispensable positionnement du bébé face à cet objet, et le décryptage de sa lutte pour l'acceptation de cet objet-mère-sein 
fantasmatique, toute bonne ou toute mauvaise dans un premier temps, puis dés-idéalisée pour être intériorisée. Travail de positionnement qui, s'il ne s'accompagnait pas d'une intégration progressive de l'ambivalence inhérent à cet objet, pouvait conduire à la fixation à l'une de ces positions primitives, la position «schizo-paranoïde» pour le psychotique. A l'hypothèse de cet autre faisant irruption, peut-être trauma, Winnicott et Bion, chacun à leur manière, répondirent. Pour Winnicott, il s'agissait d'un «cafouillage de départ», d'une «rupture de la continuité d'être » d'un individu à un moment où il était absolument nécessaire qu'elle soit maintenue. Ce qui lui permit de développer son concept du «good-enough», tout-juste-assez-adapté de l'environnement et de la «préoccupation maternelle primaire». Pour Bion, la partie psychotique de la personnalité, majoritairement agressive inhérente à chacun, nécessitait l'intervention de la «capacité de rêverie » de la mère, «ce facteur» de la «fonction alpha», version contretransférentielle de l'identification projective de M. Klein, afin de digérer les éprouvés insoutenables du bébé, et de faire barrière à la psychose.

Depuis le registre du symbolique, Lacan postula quant à lui chez le psychotique, une « soustraction dans la trame » du réseau de signifiants du sujet le conduisant à une impasse dans l'élaboration métaphorique du manque, obstruant ainsi l'accès à l'identification au tiers, «la forclusion du Nom-du-père », et aux symboles du langage. Le point commun à ces approches étant de mettre l'accent sur l'idée d'un impossible, à appréhender pour Freud, à soutenir pour Klein, à symboliser pour Lacan; et du côté de l'environnement, d'une faillite pour Winnicott et d'un défaut de contenance chez Bion.

Dans cette perspective, et afin d'étudier en quoi les modalités institutionnelles de soin nées de l'instauration, dans les années 60, de la sectorisation des soins psychiatriques, s'avèrent adaptées et pertinentes, nous nous sommes intéressé à un lieu d'accueil pour patients psychotiques, inspiré du modèle d'un Club Thérapeutique, tel qu'il fut imaginé et tout d'abord mis en place par le 
mouvement de la Psychothérapie Institutionnelle. Nous avons démarré notre recherche en posant la question d'un possible réajustement a posteriori de cette inadéquation inaugurale, due à l'inadaptation de l'environnement en termes justes, avec l'idée que c'est le cadre de soin et ce qu'il encourage d'un ré-investissement de sa subjectivité par le malade mental, qui a un rôle essentiel à jouer en tant que pourvoyeur de fiabilité, en instaurant une continuité que nous posons comme primordiale dans le projet de soin des malades psychotiques. Ce qui nous amène à introduire, corollairement à l'étude d'un dispositif fondamentalement basé sur le concept d'une transformation du malade par la transformation des rapports soignants-soignés comme principe institutionnel, la question de l'interférence des éprouvés des soignants, ou contre-transfert actif, dans la possible réinscription du malade dans une dynamique de désir. En même temps que nous essaierons d'expliquer pour quelles raisons des «actions » envers les malades «fous » se justifient, nous essaierons de montrer à travers un exemple les changements de comportement observés susceptibles de légitimer la mise en place de programmes « de soins ».

Mais retournons sur la scène psychiatrique de l'après guerre. Suite à la disparition quasi totale des aliénés de France, morts de faim pour la plupart pendant la guerre, des psychiatres se mettent à réfléchir à une remise en question nécessaire de l'Institution Psychiatrique. La question de la reconnaissance $\mathrm{du}$ «fou » comme malade mental commence alors à faire débat, et les conditions de sa probable dés-incarcération s'engagent en vue de sa réinscription sociale. C'est dans ce contexte que naît le mouvement de la Psychothérapie Institutionnelle. Il se développe dans les années 50 avec le projet d'utiliser le milieu hospitalier comme facteur thérapeutique, en faisant le pari que l'environnement du malade, le cadre où il évolue, peut servir à sa transformation dans le sens d'une évolution positive, au lieu de travailler insidieusement à sa chronicisation. La question qu'il pose est donc celle de la désaliénation de l'institution psychiatrique allant jusqu'à celle des malades, postulant qu'une partie des troubles dont souffre le malade mental est à imputer à l'institution elle-même. Ce mouvement déploiera une dynamique de mobilisation générale à 
l'égard des patients psychotiques et empruntera beaucoup à la psychanalyse. En vue de révolutionner l'Institution, il proposera une réflexion fondamentale sur la réorganisation des interrelations infirmiers-usagers. Les aliénés étant devenus des malades, les psychiatres et infirmiers psychiatriques devinrent des soignants, replaçant le respect de la personne humaine au centre des débats.

Le club thérapeutique quant à lui naquit avec les fondateurs du mouvement : Lucien Bonnafé et François Tosquelles depuis la Clinique de St Alban en Lozère, Jean Oury et Félix Guattari à la clinique de La Borde qui poursuivirent son expérience. L'idée de base était une organisation en association Loi 1901 à l'intérieur de l'hôpital, une gestion en groupe et le bénéfice d'une responsabilisation donné à des individus que leur statut de malade mental rendait a priori invalides. Sa fonction première était de sortir celui-ci de sa dépendance à la hiérarchie et de l'ouvrir à une organisation communautaire dans laquelle il partagerait un statut d'égal avec d'autres. Les membres de cette entité associative avaient pour mission l'organisation d'ateliers et la gestion des dépenses qu'ils occasionnaient. La notion d'ergothérapie est donc au fondement de ces clubs : avoir une responsabilité et en recevoir une rémunération représentait un acte thérapeutique car elle permettait d'extraire le malade de sa position passive d'objet de soin et de le placer au rang de partenaire.

Dans cette même veine, la mise en place des soins ambulatoires fut instituée dès 1959 dans le XIIIème arrondissement de Paris, à l'initiative de Philippe Paumelle. En 1960, une circulaire ministérielle officialise le «secteur». Il répond à une volonté de faire sortir le malade de l'institution psychiatrique, que sa prise en charge ne se fasse plus sur un mode asilaire mais sur son lieu de vie même, rendant ainsi possible la pratique de la prévention et le suivi à l'extérieur de l'hôpital. La loi du du 31 décembre 1985 intégrera la partition en secteurs dans une carte sanitaire générale. Chaque établissement sera chargé de gérer directement l'ensemble des activités intra et 
extra hospitalières. L'arrêté du 14 mars 1986 définit la création des hôpitaux de jour, centre médicaux psychologiques (C.M.P), centre d'accueil thérapeutique à temps partiel (C.A.T.T.P.), services d'hospitalisations à domicile, tous dotés d'équipe de psychiatres, psychologues, infirmiers psychiatriques.

A partir de cette brève présentation historique et théorique, la ligne directrice qui nous servira de guide dans l'écheveau des références dont nous nous réclamons serait alors : si un cafouillage de départ, une fixation, a conduit à une impasse dans la construction psychique, comment se justifie un accompagnement conçu pour faire des malades souffrant de troubles psychiatriques des sujets de leur présent comme de leur devenir. Par ailleurs, s'il y a forclusion, subsiste-t-il encore pour ces sujets la possibilité de se saisir d'espaces potentiels ouvrant la voie d'une évolution, à défaut d'une guérison au sens strict du terme. Si à un certain moment, quelque chose est venu à manquer, peuton postuler qu'une élaboration ultérieure pourrait permettre non pas de remplacer mais de tisser autour de ce qui avait fait vide, absence, de travailler cette rupture de continuité, de re-solliciter cette relation libidinale défaillante à la réalité, de redonner corps à ce qui ne s'est pas maintenu, de ré-injecter du symbolique à la place du réel. Le cadre de soin est-il en possession des virtualités capables de remodeler cette continuité brisée, par la variété des situations qu'il offre et la multiplicité des expériences à tenter en fonction des patients et de leurs histoires ?

Le B., association créée par des infirmiers psychiatriques d'un C.A.T.T.P. et attenante à celui-ci, est inspiré du club thérapeutique. Il constitua le décor de cette recherche et le berceau de mes réflexions. C'est une maison de ville en plein Paris dont la pièce principale commune est aménagée comme un café. Un patient-adhérent se tient au comptoir. Souvent aux murs, des photos d'expositions organisées par l'association. Le bar est un lieu stratégique. De derrière lui, on embrasse l'ensemble de la salle. Celui qui en est responsable occupe une place de choix. Car le fait de devoir assurer la gestion des gains est une responsabilité que l'équipe accorde à un adhérent. 
Les personnes qui le fréquentent sont des patients souffrant de troubles psychiques, psychotiques pour la plupart. Pour s'y rendre, ils payent une cotisation qui fait office d'adhésion. Après cette formalité, leur statut change : il deviennent «adhérents » au même titre que toutes les personnes fréquentant le lieu, soignants inclus. Nous insistons sur le fait que la vocation officielle de ce lieu n'est pas d'être un lieu de soin, ni de consultation, on ne s'y rend pas sur indication médicale, on n'y vient pas chercher le savoir de celui supposé le détenir. C'est avant tout un espace informel de rencontre. Comme le disait J. Oury : «c'est un lieu où on va »... un peu comme dans un café, de là probablement son nom de «café-club». On y joue aux cartes, à des jeux de société, on boit un verre (sans alcool), on lit le journal, discute. C'est donc autant un lieu de passage qu'un lieu où il est possible de s'inscrire dans une continuité.

Nous commencerons par quelques remarques issues d'une expérience avec un patient, qui nous a semblé constituer une illustration de la reprise d'un processus psychique. Nous avons souhaité en rendre compte, car elle comporte à notre avis des éléments qui laisseraient à penser que les activités proposées dans ce cadre sont initiatrices de mouvements re-dynamisants, et pourraient engendrer une sorte de re-entrée dans des relations libidinales. Dans tous les cas, cette description constitue un témoignage de ce qui peut être considéré comme un cheminement en commun dans un objectif de soin.

\section{A l'atelier cuisine :}

C'était la première fois que je participais à cet atelier où, psychologue en herbe, j'avais à r-animer, un groupe de patients psychotiques considérés plus difficiles. Avec Olivier - à qui je donne le nom de l'arbre dont les rameaux secs et noueux ne laissent en rien présager la richesse des fruits qu'ils porteront -les questions s'amoncelaient. Lors de notre première entrevue, j'étais assez intimidée. Il m'avait été décrit comme un patient dont on n'attendait plus grand chose. Il était assis, bavait, se mouchait dans ses vêtements et délirait à ses heures. Certains patients lui jetaient des remarques : 
«t'es vraiment dégueulasse, tu pourrais te moucher ». Il arborait pourtant souvent des nouveaux vêtements qu'on ne lui connaissait pas : «trouvé dans les poubelles », nous disait-il, perdait ou échangeait contre des cigarettes ceux que lui achetaient ses parents. Le troc de cigarettes l'occupait beaucoup, en témoigne la fois où, hospitalisé à maison Blanche, il en avait échangées contre sa carte d'invalidité. Je fus dès le premier jour l'objet d'un transfert massif, me dirent les infirmiers. «Massif », comme le diagnostic de sa psychose... - «psychose massive » était-il écrit dans son dossier, deux feuilles recto dans le bureau des infirmiers... pour 20 ans de suivi, - ils n'avaient pas vu Olivier comme ça depuis bien longtemps, enjoué, et même bavard. Le sourire, sorte de rictus d'allure terrifiante dont il me gratifia le premier jour, laissant voir une dentition considérablement décatie, altérée et noircie semblait-t-il, autant par les traitements que par l'abus de nicotine, fut largement salué par tous comme le signe d'un «mieux » d'Olivier. C'était la seule fois qu'on le voyait sourire depuis qu'il fréquentait le C.A.T.T.P. Ce serait également son seul sourire en six mois. Connu des services, très «régressé», il fréquentait le groupe cuisine depuis plusieurs années, et scandait ses venues de propos stéréotypés : «en 2004, 2005, 2006, je vais à la piscine et je fais la cuisine au CATTP. En 2007, 2008, je fais que la cuisine. » Il ajoutait parfois en fin de repas quand je lui demandais de faire la vaisselle : «en 2004, 2005, 2006, je fais la vaisselle, en 2007 et 2008, se saisissant prestement de l'éponge, je nettoie la table et je balaye ». Je le trouvais drôle...

Les jours ou «ça n'allait pas », il nous lançait : «j'en ai marre du CATTP, j'veux plus rien foutre », et appuyait ses propos par une sortie séance tenante de la cuisine, nous laissant pantois face à cet intangible refus. Contraint et forcé, il nous accompagnait pourtant faire les courses, non sans nous rappeler, se baissant pour ramasser les mégots écrasés - ce que nous lui recommandions fortement d'éviter de faire - les allumant et les consommant le plus avidement qu'il pût, qu'il était un cas plutôt désespéré. Je me mis à le solliciter fréquemment car il me semblait être réceptif à ces sollicitations. D'autant plus qu'avec d'autres elles avaient moins de succès... En présence d'Olivier, 
s'effectuait en moi une sorte d'accordage intuitif. J'avais l'impression de ne faire que peu d'efforts, excepté celui de me brancher sur son propre canal. Je me sentais happée par lui, comme répondant à quelque ultra son ou mue par une télécommande invisible qui m'enjoignait de m'accorder à lui. Olivier me déplaisait pas, n'éveillait pas en moi la moindre agressivité. Je ressentais en sa présence une disponibilité décuplée, en témoigne l'intérêt que je me découvrait pour lui, et l'espace que je découvrais de pouvoir accueillir une demande que je supposais émaner de lui, que je pourrais dire avoir fait jaillir de l'implicite, sans pour autant disposer du moindre argument pour l'affirmer. Car, n'est-ce pas ce que ce patient m'inspirait : abandon, sclérose psychique, perte de communication, pitié même face à sa légère incurie et les réactions qu'elle suscitait, qui m'a fait croire, imaginer, soupçonner et finir par entrevoir une modification de son comportement. Laquelle de ces deux motions, l'amélioration, ou mon désir de l'amélioration précéda l'autre, je ne saurai le dire.

Pourtant, le temps que je passais à requérir de lui quelque participation était toujours payé en retour du temps qu'il accordait dans l'exécution de la tâche requise : couper des pommes, remuer la pâte à crêpe, battre en neige les blancs, était séance après séance, accepté...attendu...? Un jour, où il s'était montré ostensiblement intéressé par l'idée de m'accompagner à la boucherie, il me parla de lui, de sa vie, me/se raconta : son foyer, son amie comme il l'appelait, sans que je lui pose de questions. J'étais spectatrice d'un déploiement de la capacité, ignorée, d'Olivier, à s'ajuster. Olivier, qui répétait les même phrases et qu'on aurait dit figé dans la pathologie, dans un même comportement décalé, s'ajustait à nous, à chaque séance, séance après séance, racontait, en réponse à un : «Alors Olivier, comment ça va aujourd'hui ?» à peine appuyé, des anecdotes toujours nouvelles. Lorsqu'on sait que la difficulté d'ajustement est un trait caractéristique de la pathologie psychotique, je me demandais dans quelle mesure la fréquentation de l'atelier cuisine et la possibilité qu'il semblait admettre que sa parole soit reçue n'avait pas ouvert une brèche dans le rituel de son comportement. J'essayais de comprendre... Il ne se trouvait pas dans une position de malade à faire valoir, à conserver comme son seul bien face à celui supposé savoir quelque chose de plus que lui sur sa maladie. Ici, il n'était pas dans une démarche de demande de soins. Il était 
surtout là parce qu'il devait s'occuper un peu pour pouvoir demeurer dans le foyer dans lequel il vivait depuis qu'il avait du quitter l'hôpital de jour en raison de sérieux troubles du comportement. Dans l'atelier, je le ressentais comme bénéficiant d'un effet hors cadre inhabituel : je passais du temps à l'inciter à occuper une autre place, n'étant pour lui ni «le médecin », ni le personnel encadrant habituel du centre, n'appartenant pas à sa communauté (il était juif de famille pratiquante) organisée suivant des règles strictement instaurées. Je me disais qu'il fallait tenter quelque chose pour l'inciter à s'exprimer depuis un endroit qui serait le sien en tant que sujet. D'un patient bavant et peu fiable dans la confection de la recette, il était devenu incollable sur l'heure à laquelle arrêter le four, enlever la mousse au chocolat du freezer, parfois presque intarissable : «quand je viens ici, je dis tout ce que j'ai à dire » déclara-t-il un jour comme pour expliciter et traduire ce que je m'évertuais à réfléchir et n'osait supposer en conséquence de son investissement de plus en plus affirmé : il avait envie de parler de lui. Sa réceptivité à l'autre, insoupçonnable au premier abord, me stupéfiait tout en m'envahissant. Il n'était plus cet objet potiche, ce bouche trou, ce bouche angoisse, toujours à sa place, obstruant l'entrée à toute interrogation : rassurant pour tous. Car j'avais remarqué que sa nouvelle éloquence avait également pour effet de déplacer la dynamique ambiante. Son état faisait l'objet d'interprétation : «il est angoissé, disait-on, sinon il ne parlerait pas comme ça... » Je me demandais qui était le plus angoissé... Je me suis alors questionnée sur ce que, depuis ma place, je pouvais faire, pour que, s'il y avait un quelconque espoir qu'il se déplace, j'amorce quelque chose dont il puisse se servir... Je suis donc allée consulter son dossier au C.M.P. dont il dépendait et où il consultait régulièrement un psychiatre. En vingt ans de suivi, je ne trouvais que peu d'informations le concernant, excepté qu'il était considéré comme un «psychotique hébéphrène stabilisé sur un mode déficitaire ». J'ai tenté de soulever son cas en réunion d'équipe, mais son cas se perdit au détour des obligations administratives...

Pour clôturer l'année, nous nous sommes retrouvés un midi au restaurant. Je ne sais s'il était triste, 
moi je l'étais c'est sûr. Est-ce en guise d'adieu qu'en ce dernier jour d'un stage qui m'avait apporté tant de matière à penser les patients, Olivier me fit un cadeau. Il était parti fumer à l'extérieur du restaurant. Il revint soudainement, me regarda et : «un miracle s'est produit me dit-il, j'avais dix cigarettes, je n'en ai plus que huit... » et il se mit à rire, un long rire qui sembla lui-même le surprendre comme s'il s'y essayai pour la première fois. Et je m'en allai, emportant avec moi le rire d'Olivier miraculeusement surgi de son délire et dont l'écho accompagne toujours mon travail aujourd'hui... A l'atelier cuisine, Olivier s'était inscrit dans un cadre invisible, qui en était tout de même un sans qu'il s'en rende compte, et auquel il se laissait prendre. Je ne pourrais me prononcer sur une éventuelle amélioration ni sur les raisons de cette amélioration, mais je pense pouvoir dire que sa parole s'était déliée. En offrant la possibilité aux patients de mener quelque chose de bout en bout par soi-même, outre la possibilité d'une re-narcissisation réelle dans certains cas, l'atelier thérapeutique m'a semblé être l'occasion d'assister, d'être le témoin autant que l'acteur d'un processus à l'oeuvre, d'un aboutissement, mais également de pouvoir profiter (manger, consommer, jouer, chanter) d'un résultat. A la cuisine, il fut l'occasion de traverser plusieurs étapes de la gestation à l'éclosion, en incluant sa propre participation. De bout en bout, le patient serait là, de la préparation jusqu'au moment de manger qui est autant détruire oralement qu'incorporer le «bon à manger », c'est-à-dire le «bon » tout court, ce moment de ré-création commune, de participation, cette forme de jeu, qui se termine mais reprend chaque semaine. Absorber, détruire, et recommencer : le détruit-retrouvé de Winnicott. Sans oublier que ce travail s'accompagne de paroles, une parole qui s'insinue dans les espaces ouverts et qui éclot dans la situation du faire. Mener à la parole, en être le secrétaire et le témoin, c'est là que j'ai pu situer ma fiabilité et ma capacité de contenance.

\section{$\underline{\text { Discussion }}$}

Ce que sous-entend l'idée d'un lieu fonctionnant hors indication thérapeutique, est qu'il puisse être utilisé par le patient à sa guise, selon son bon vouloir comme le dit l'expression française, qui dit 
bien à sa manière l'implicite contenu dans cette notion d'utilisation.

Cette latitude constitutive nous permet d'oser un parallèle avec ce que décrit Winnicott sur l'utilisation de l'objet, c'est-à-dire une modification de la position du sujet par rapport à celui-ci, modification porteuse de transformations pour le sujet lui-même par la transformation de son rapport à l'objet utilisé.

A la base de la théorie de Winnicott de l'utilisation de l'objet, il y a le développement de la capacité à le faire, celle-ci faisant partie du processus de maturation, lui-même dépendant d'un environnement facilitant. Pour pouvoir utiliser l'objet, le sujet doit pouvoir le détruire, c'est-à-dire pouvoir le situer hors de son contrôle omnipotent. Si dans la psychose telle qu'il la situe, il y a un cafouillage de départ, une rupture irrécupérable de continuité, l'objet n'a pas prouvé sa fiabilité, n'a donc pu être détruit et n'existe pas en quelque sorte. Le cadre du B. est d'emblée posé comme pouvant être remis en question par le sujet, puisque c'est à lui qu'il revient en premier lieu de le faire exister, par sa présence, la régularité de ses venues, sa participation aux activités. On peut peut-être dire que sa particularité réside dans le fait qu'il n'existe justement que dans la mesure où le patient parvient à l'utiliser, c'est-à-dire à traverser les étapes (y survivre) par lesquelles il passe d'un état où il est en relation avec lui à celle où il devient capable de l'utiliser, c'est-à-dire de le reconnaître différencié, de se positionner par rapport à lui. La forme du $B$. exige sans cesse du patient qu'il fasse des choix, et en cela on peut avancer que ce qui est demandé au patient c'est de refaire vivre l'objet $B$. à chaque fois qu'il est en relation à lui, puisque tout ce qu'il y fait, il ne le doit qu'à sa propre décision, c'est de son initiative qu'il s'y rend, y exerce son activité, ou même n'y fait rien de spécial.

En déplaçant le sujet d'une position où il dépend de l'institution pour ses soins, pour l'image que celle-ci lui renvoie de lui au travers du diagnostique qu'elle en fait, du traitement qu'elle lui prescrit, des rendez-vous qu'elle lui donne, et en lui offrant la possibilité de devenir agent du 
processus auquel il participe par la fréquentation de cet endroit, ne se présente-t-il pas comme un modèle d'objet à utiliser ? Peut-on alors émettre l'idée qu'il se place en position d'aire tierce d'expérience, cet espace potentiel ni moi, ni non-moi, berceau d'une intégrité psychique en devenir et de possibilités de créativité ?

Parallèlement à l'offre d'investissement libre de ce lieu, nous souhaitons mettre l'accent sur le mouvement de va et vient qu'il octroie entre l'intérieur et l'extérieur, le dedans et le dehors et qui pourrait d'après nous lui permettre de fonctionner symboliquement comme un espace transitionnel. Ce concept concerne «le chemin parcouru par l'enfant lorsqu'il passe du subjectif pur à l'objectivité [...] ce que nous percevons de ce cheminement progressif vers l'expérience en train de se vivre ». ${ }^{1}$

Cette théorie nous sert de bâton sourcier pour explorer le fonctionnement de ce « club » à vocation thérapeutique et ce qu'il encourage. Car, contrairement à ce que les substantifs espace et objet laissent à penser, il ne s'agit pas avec l'aire transitionnelle d'une entité délimitée mais au contraire d'un processus maturatif. Dans la théorie de Winnicott, l'enfant est dans l'illusion que l'objet qu'il trouve là juste à sa disposition c'est lui qui l'a créé, et cela, grâce à une «good enough mother », c'est-à-dire tout-juste-assez-adaptée à son enfant. Progressivement, c'est grâce à cette même mère que s'effectue ce travail de désillusion et d'acceptation de la frustration qui, pour Winnicott, est à la base de la possibilité de l'enfant de reconnaître les objets comme rééls, c'est-à-dire haïs autant qu'aimés. La mise en place de ce dispositif de continuité, l'illusion continue de l'aire transitionnelle, permet à ce qu'une relation s'établisse entre l'enfant et le monde. C'est de ce même mouvement dont parle Winnicott lorsqu'il explique qu'il s'agit d'une illusion qui permet à l'individuation de se produire.

1 WINNICOTT D.W. (1971) : «Objets transitionnels et phénomènes transitionnels » in Jeu et réalité, Paris, Gallimard, $p$ 175. Citation originale : [...] Nous avons préféré citer la première traduction de Claude Monod et J-B Pontalis de l'édition Gallimard de 1975 de ce passage, à celle de l'édition Folio de 2006 (cf bibliographie) où il est écrit : «le voyage qu'accomplit le petit enfant et qui le mène de la subjectivité pure à l'objectivité (...) ce que nous percevons du voyage qui marque la progression de l'enfant vers l'expérience vécue », p 36. 
Si, pour Winnicott, l'aire transitionnelle est ce qui, en autorisant les va-et-vient moi/non-moi du bébé au niveau psychique, lui permet de se confronter à la réalité, alors, le caractère ouvert du B., les passerelles qu'il installe, les va-et-vient qu'il provoque, sont peut-être à même d'abriter un processus d'expérimentation et pourquoi pas de reconstitution d'une intégrité psychique qui fait défaut au patient psychotique, en lui apparaissant sous la forme d'un lieu à investir dans une totale liberté et où progressivement il devient capable de supporter un rapport à la réalité jusqu'alors marqué par la conflictualité.

Il nous semble possible de voir, dans cette proposition d'aire transitionnelle appliquée à l'endroit, une alternative « in progress » à ce mouvement d'effilochage de soi, de destruction interne, d'où faire émerger un premier moi juste avant qu'il soit capable d'utiliser l'objet. C'est dans ce fonctionnement en aire tierce qu'est contenue la potentialité d'un lieu thérapeutique, d'investissement ou de ré-investissement au sens où quelque chose du patient peut se reconstruire, se réincarner, notamment grâce aux ateliers qui, en encourageant la créativité, se superposent à ce processus de maturation et lui confèrent la possibilité d'en opérer une traduction simultanée dans le symbolique. Le B., un lieu, des heures fixes, des personnes encadrantes, et pas seulement des adhérents livrés à eux-mêmes, un « objet fiable », qui se dévoile à mesure qu'on l'investit, comme on s'investirait soi, progressivement, dans un mouvement de découverte et de construction de son propre moi, un lieu où l'on repère certaines balises fixes : des horaires, des activités, une équipe de soignants que l'on connaît, à l'inverse d'un lieu qui s'autogère et où rien ne se prévoit. Mais de l'autre, il apparaît également comme un endroit traversé de multiples possibles, échanges, activités nouvelles. Celles-ci peuvent d'ailleurs être décidées rapidement, à l'initiative d'un patient, d'un soignant, d'un stagiaire de passage. Car ici, tout a été pensé de manière à être continuellement réinterrogé, ce qui donne au déroulement du temps un aspect non permanent, non ritualisé.

A l'encontre de la léthargie, là où le patient court toujours le risque de succomber à la menace 
d'identification au cadre institutionnel, il lui ouvre une voie qui est exactement le contraire de l'enlisement dans la chronicité. Lorsque le cadre n'est pas figé, il évite que s'instaure ce mouvement de fossilisation dont il est souvent question avec les psychotiques. Et, c'est peut-être en déjouant ce mécanisme qu'il met au travail. Le cadre aurait dans cette optique une portée élaborative. Il abriterait une dynamique créée par les sollicitations faites aux patients, en contiendrait les débordements éventuels tout en se protégeant d'un mouvement répétitif étouffant toute émergence de vie. Un tel travail permettrait que le malade en vienne lui-même à se penser autrement par le regard différent porté sur lui et, d'une image dans laquelle il est enfermé, il serait possible d'initier un mouvement inverse de déstigmatisation.

Ce développement nous conduit à situer le cadre, qui, bien que perçu comme une instance qui limite, délimite, appose des frontières - autorisant des processus d'émergence depuis l'informe fonctionne également dans la dialectique de l'entrer/sortir, plein/vide, dedans/dehors, dans une intelligence des limites qu'il a lui-même pourtant induit. Et si des réactions plus primaires d'agressivité s'y donnent à voir, on s'attend à ce qu'elles surviennent. C'est d'ailleurs parce qu'il est indispensable de les contenir que la présence des soignants s'avère essentielle. Si en effet, il est donné la possibilité au patient d'utiliser le cadre comme un objet, c'est-à-dire de le détruire en fantasme, cela signifie que cet objet doit pouvoir contenir les réactions, débordements qu'il induit. On peut donc également supposer que le cadre fonctionne comme un lieu de digestion de cette agressivité. Nous souhaitons appliquer à cet exposé la théorie de la contenance déployée par Bion. C'est au moment précis où il souligne l'envahissement de la partie psychotique de la personnalité par des mouvements agressifs qu'elle ne peut intégrer, interdisant toute potentialité d'accès à la pensée, au symbolique insoutenable et donc nié, qu'il fait appel à une capacité extérieure pour se réapproprier tout ce qui l'a débordé et qu'il a expulsé hors de lui. Bion pose que la fonction alpha, dans un mouvement de va-et-vient mère-enfant, correspond à une certaine capacité de la mère de recevoir et de transformer les sensations émotionnelles trop violentes pour le bébé, de « digérer » à 
sa place ces éléments inélaborables pour ensuite les lui restituer : la capacité de rêverie de la mère, «un état d'esprit réceptif à tout objet provenant de l'être aimé ». C'est grâce à elle que ces impressions trop douloureuses pourraient être réintégrées par le bébé et acquérir le statut «d'éléments alpha», la prolifération d'éléments alpha produisant une barrière de contact permettant au bébé de se construire un rempart de protection sur lequel il s'appuiera pour construire sa différenciation psychique et accéder à sa propre capacité de penser.

D'une certaine manière, le fait que l'on se trouve dans un lieu contenant, écoutant, voire maternant invite à la régression. Si on accepte l'hypothèse que la psychose provient d'un effondrement à un stade très précoce du développement, alors il est très important d'accueillir la régression du patient psychotique et de lui donner le sentiment que face à la crainte de l'effondrement quelque chose sera là pour l'accueillir. Comme l'écrit Maud Mannoni, il ne s'agit pas forcément de soigner la psychose, mais de «la recevoir», de « recevoir l'effondrement». C'est peut-être à cette condition que peut s'instaurer la confiance nécessaire dans les liens et que s'ouvrira la possibilité pour qu'un espace psychique se construise. C'est aussi à cette condition que la parole s'autorise, une parole libre, hors rapport médecin-malade, une parole surprise, prise sur le vif et facilitée par les nombreux transferts qu'induisent les présences variées. Ces transferts, poly-objectaux, souvent activés dans les ateliers qui sont le creuset des moments où ça parle.

Quels sont les rapports entre la contenance et le cadre ? Nous sommes partis de l'idée que le cadre était contenant. Mais il apparaît à la lumière de nos observations que ce n'est pas le cadre qui définit la contenance, que celle-ci peut être située dans un au-delà du cadre. On en arrive à ne conserver que la notion de contenance elle-même, à la faire émerger comme un processus subjectif qu'autorise le cadre mais qui ne lui est pas co-extensif. Il l'invite, il la suppose, il la sollicite, il cherche à l'accueillir, on peut même dire qu'il la féconde. Et au final, nous aboutissons à cette conclusion que c'est à proprement parler plutôt la contenance qui fait cadre et non l'inverse. 


\section{$\underline{\text { Conclusion }}$}

A partir de l'hypothèse d'un lieu/cadre de soin susceptible, postérieurement à la proposition théorique d'une faillite initiale de l'environnement, d'oeuvrer à la reconstruction ou à la réélaboration de ce cafouillage, de ce chaînon manquant au psychisme des patients psychotiques, nous avons souligné l'importance de créer un espace de convivialité, un lieu de vie qui ne soit pas un lieu de soin, dans le but de lutter contre l'aliénation sociale, de favoriser les échanges sociaux et les intérêts culturels. Le club thérapeutique, en proposant un fonctionnement associatif tourné vers la créativité, la responsabilisation et la resocialisation de malades, opérant comme un outil indispensable de ce dispositif. Ce travail permettait une vue d'ensemble, d'embrasser la totalité des échanges et mouvements, tout en saisissant beaucoup de points de détails d'un seul coup, en bref, d'être dans le vif du sujet et loin des méandres institutionnels.

Nous avons également envisagé de discuter la disposition particulière des soignants dans la reprise de la dynamique de vie des patients psychotiques, mais ce point mérite une accentuation spécifique que nous nous proposons d'aborder en profondeur dans un article ultérieur.

Pour l'instant, nos observations nous permettent de mettre en lumière certains points de réflexion relatifs au dispositif étudié et en lien avec notre hypothèse : la nécessité du maintien d'un milieu non aliénant capable de faciliter les échanges inter-humains, une offre d'utilisation de ce lieu comme objet et la possibilité qu'elle ouvre vers la secondarisation, la place ainsi octroyée à l'émergence d'un espace psychique, la reconquête d'un statut d'acteur de sa propre vie susceptible de relancer la dynamique libidinale, la production d'une contenance comme conséquence d'une implication active des acteurs-soignants. Il nous semble que dans cet espace, la multiplicité de possibilités de rencontre, de relations, sont autant de possibilités de transfert, de rechargement au niveau des pulsions de vie, en déficit chez le psychotique. En cela, ce que le dispositif offre de continuité nous paraît essentiel dans les progrès qui peuvent s'amorcer dans l'état du patient. Cette 
continuité se marque particulièrement dans cette contenance que nous évoquions, une disposition omniprésente, que favorise l'investissement particulier des soignants en ce qu'il se source à des motivations, qu'elles soient conscientes ou inconscientes, à soutenir et à accompagner tout ce qui se laisse voir, entendre, remarquer d'impulsions en direction d'un reprise d'un mouvement vital.

Nous avançons que le travail que sous-tend ce type d'espace conduit à une ré-appropriation de son statut d'individu, souffrant psychiquement mais pas pour autant inadapté ou incivil. Ce qui constitue déjà à lui seul un glissement pouvant s'avérer fécond s'il introduit à s'autoriser l'assomption de choix allégée du poids de la stigmatisation. Et qui sait si ça ne déboucherait sur de réelles transformations. Nous rejoignons Bion lorsqu'il écrit : «il y a lieu de s'attendre à ce que le schizophrène parvienne à sa propre forme d'ajustement à la réalité, qui bien qu'elle ne ressemble pas à celle que peuvent atteindre des patients moins perturbés, n'en est pas moins digne du titre de «guérison $~^{1}{ }^{1}$. A ce titre, la question d'un regard autre porté sur et par le patient lui-même peut être considéré comme une transformation à part entière.

Ce point nous permet de rejoindre la question de l'éthique, car le travail avec des patients souffrants de troubles psychiatriques, s'il en appelle à réfléchir à l'importance du sentiment de continuité interne, devrait également inclure cette même dimension dans le traitement adapté à ces personnes, c'est-à-dire un suivi dans la durée. Ce qui impliquerait alors que l'on n'inscrive plus la question du soin aux patients psychotiques dans la marge qui sépare le normal du pathologique, mais que l'on en vienne à considérer ces troubles comme une enclave pathologique au sein du normal, dont la survenue est due aux aléas de l'existence, qui s'appréhende comme une donnée à laquelle s'adapter - et en cela tous les moyens thérapeutiques sont les bienvenus - et non comme une déviation à éradiquer.

1 BION W.R. (1953). « Notes sur la théorie de la schizophrénie », in Réflexion faite, p 42. 
Concernant la question cruciale d'une éventuelle transformation psychique des patients, on a montré que cette tentative, si elle ouvrait des voies de déplacement des sujets par rapport à leur état, leur parole, pouvait aller jusqu'au désamorçage des processus internes conduisant à la crise et à l'hospitalisation. L'interrogation corollaire et essentielle qu'elle soulève d'après nous concerne le fait de savoir si les patients réussissent à utiliser ce qu'ils ont acquis/développé/compris uniquement dans le cadre de ce lieu. Ou bien, le fait d'être baigné dans cette dynamique extrainstitutionnelle et tout ce qu'elle comporte comme écarts avec la manière dont on traite le psychotique dans les services psychiatriques généraux à grand renfort de neuroleptiques, permet-il à quelque chose de se développer et de suffisamment s'inscrire et s'intérioriser au niveau psychique chez le patient pour qu'on soit autorisé à parler de réelle portée thérapeutique. 